\title{
MODELO DE LEILÃO MULTIPERÍODO PARA SISTEMAS HIDROTÉRMICOS COM REPRESENTAÇÃO DA TRANSMISSÃ̃O
}

\author{
JULIO C. BREDA ${ }^{1}$, LEONARDO NEPOMUCENO ${ }^{1}$.
}

\author{
1. Laboratório de Pesquisa, Otimização e Estudos Econômicos em Sistemas de Potência, Departamento \\ de Engenharia Elétrica, Universidade Estadual Paulista - UNESP \\ Endereço para Correspondência \\ E-mails: vandenbreda@yahoo.com.br, leo@feb.unesp.br
}

\begin{abstract}
This paper proposes a network constrained multiperiod electricity auction model for hydrothermal generation systems for the context of the day-ahead energy markets, which incorporates the transmission congestion and losses in network as well as nonlinearities and nonconvexities associated with generation system. Some innovative aspects are introduced in the model, such as: i) the hydraulic generation is optimized through the offer cost function proposed; ii) interrelationships between pool and bilateral markets are represented through a single optimization problem; iii) future deficit risks are intrinsically mitigated by adopting daily energy targets; iv) the model calculates the market clearing prices on an hourly basis for each bus/region (nodal spot prices) and show a coherent correlation between hydrological conditions and the calculated prices; v) the representation of the network transmission in a single optimization problem, does not require ex post heuristics procedures to allocate the network losses, nor does require any power flow congestion management procedure; The proposed model is solved by a primal-dual interior point method and is evaluated by simulations involving a test system. The results are focused on sensitivity analyses involving the parameters of the model, in such a way to emphasize its main modeling aspects. The results show that the proposed model is able to calculate short-term nodal prices for the day-ahead markets of hydrothermal power systems and show the influence of the transmission network in the price formation.
\end{abstract}

Keywords—Energy Markets, Optimization, Transmission loss and congestion.

Resumo — Este trabalho propõe um modelo de Leilão Multiperíodo para Sistemas Hidrotérmicos com Representação da Transmissão para o ambiente de mercados de energia do dia seguinte, que incorpora o congestionamento e perdas na transmissão, bem como não-linearidades e convexidades associados com o sistema de geração. Alguns aspectos inovadores são introduzidos no modelo, tais como: i) a geração hidráulica é otimizada através da função de custo de oferta de energia proposta; ii) inter-relações entre mercados pool e bilaterais são representados através de um único problema de otimização; iii) riscos de deficit futuro são intrinsecamente mitigados através da adoção de metas diárias de geração, iv) o modelo calcula os preços de equilíbrio de mercado em base horária para cada barra/região (preços spot nodais) e mostram uma correlação coerente entre as condições hidrológicas e os preços calculados, v) a representação da rede de transmissão em um único problema de otimização não necessita de procedimentos ex post e heurísticas para alocar as perdas e os congestionamentos na transmissão. O modelo proposto é resolvido pelo método de ponto interior primal-dual barreira logarítmica e é avaliado por meio de simulações que envolvem um sistema de teste. Os resultados estão focados na análise de sensibilidade envolvendo os parâmetros do modelo, de modo a realçar seus principais aspectos. Os resultados mostram que o modelo proposto consegue calcular os preços nodais de curto prazo para os mercados do dia seguinte de sistemas hidrotérmicos e mostram a influência da transmissão na formação de preços.

Palavras-chave — Mercados de energia, Otimização, Perdas na transmissão e congestionamento.

\section{Introdução}

A reestruturação do setor elétrico no mundo, decorrente da implantação dos mercados de energia, tem levado a importantes discussões e alterações nos modelos de planejamento e operação dos sistemas elétricos, antes fechados ao capital privado, passaram com o surgimento dos mercados de energia, ao fomento à competição e à livre iniciativa. A tarefa de despachar a geração e calcular os preços da energia, antes realizada, no ambiente regulado, pelo despacho de geração, utilizando-se modelos de unit commitment, despacho ou pré-despacho de geração (Soares, Salmazo, 1997), é agora feita por modelos de leilão de energia (Arroyo, Conejo, Galiana, Motto, 2002), (Conejo, Arroyo, 2002).

Os modelos de leilão para os mercados de energia de curto prazo ou do dia seguinte, têm sido implementados na maioria dos mercados de energia estabelecidos, nos quais agentes geradores ofertam venda de energia e consumidores fornecem lances de compra de energia através do leilão realizado no mercado pool para cada hora/fração do dia seguinte. As ofertas de venda e os lances de compra são avaliados pelo Operador de Mercado (OM), o qual utiliza um modelo de leilão, que tem a tarefa de definir quais as ofertas/lances serão aceitos. Essa definição é feita com base em uma função objetivo do problema (Arroyo, Conejo, Galiana, Motto, 2002), (Conejo, Arroyo, 2002) , (Luh, Blankson, William, Cheng, Yan, Stern, Chang, Zhao, Feng, 2006), e sujeito às restrições associadas à operação e ao planejamento dos sistemas de geração e transmissão.

Apesar de haver um grande número de trabalhos na literatura propondo diferentes metodologias e estratégias para ambiente de mercados de energia (Motto, Galiana, Conejo, Arroyo, 2002) , (Carrión, Arroyo, 2006), estes trabalhos abordam modelos predominantemente termelétricos. Tais modelos também não incorporam a representação das perdas e congestionamento na transmissão embutidos internamente ao modelo de leilão, deixando a cargo de ajustes, heurísticas e correções ex post a incorporação das perdas na transmissão e do congestionamento do sistema. 
Alguns autores têm criticado a maneira discriminatória desses modelos (Motto, Galiana, Conejo, Arroyo, 2002), (Conejo, Arroyo, 2002), (Conejo, Galiana, Arroyo, García-Bertrand, Chua, Huneault, 2003), já que a presença desses ajustes podem produzir distorções econômicas. Mostram-se ainda, que os preços calculados por modelos de leilão termelétricos que ignoram a transmissão, deixam de computar as parcelas dos preços associadas ao congestionamento e às perdas na transmissão. Os autores mostram em (Conejo, Galiana, Arroyo, García-Bertrand, Chua, Huneault, 2003) que a associação em cascata desses ajustes heurísticos nos preços podem produzir preços significativamente diferentes dos preços de equilíbrios reais, além de levar a ineficiências econômicas e a formação de subsídios cruzados.

Seguindo a mesma linha de raciocínio adotada para os sistemas com geração puramente termelétrica, a utilização de ajustes e correções ex post devem distorcer os preços de equilíbrio de energia de sistemas hidrotérmicos, e podem levar a ineficiências econômicas e subsídios cruzados indesejáveis no mercado. Exemplo disso é o modelo clássico de leilão hidrotérmico, adotado pela Noruega (Fosso, Gjesvilk, Haugstad, Mo, Wangensteen, 1999), no qual a função de bem estar social é minimizada em um problema monoperíodo escrito para cada intervalo de tempo, separadamente, e sem a representação da transmissão. Para lidar com as restrições operativas intertemporais, com as não linearidades, não convexidades associadas ao subsistema de geração, e ainda, para lidar com os congestionamentos e perdas na transmissão, a solução do problema deve sofrer uma série de correções $e x$ post.

No entanto, apesar dos diversos modelos de leilão existentes, ainda falta um modelo eficaz, que leve em consideração sistemas elétricos com geração predominantemente hidrelétricos, como é o caso do Brasil. Desse modo, este trabalho propõe um modelo de leilão hidrotérmico que incorpore restrições intertemporais ao problema de leilão hidrotérmico, formulando-o através de um modelo multiperíodo, ao invés de uma série de modelos monoperíodo individuais. Além disso, esse modelo incorpora a representação da transmissão internamente ao leilão, de modo que, não existe a necessidade de etapas $e x$ post nem para resolver problemas associados a restrições intertemporais, nem para resolver problemas associados ao congestionamento e às perdas na transmissão. A formulação proposta evita as ineficiências econômicas e os subsídios cruzados, fornecendo preços de equilíbrio e despachos mais representativos.

Para solução do Leilão Multiperíodo para Sistemas Hidrotérmicos com Representação da Transmissão (LMSHRT) foi utilizado o Método de Pontos Interiores Primal Dual Barreia Logarítmica (MPI) (Wright, 1997) que apresenta uma alta eficiência computacional e tem sido amplamente utilizado para solução de problemas de otimização de grande porte.

\section{Descrição do Modelo Proposto}

O modelo LMSHRT proposto (1), busca minimizar os custos de oferta de energia das usinas termelétricas e hidrelétricas $(1 a)$, respeitando as restrições operativas e de mercado. Essas restrições dizem respeito ao atendimento da demanda para cada barra/região do sistema $(1 b)$, o atendimento das metas energéticas para usinas hidráulicas $(1 c)$, o atendimento do montante dos contratos bilaterais firmados entre os consumidores e os agentes geradores termelétrico (1d) e hidrelétricos (1e), atendimento dos limites mínimos e máximos de geração em cada usina termelétrica (1f) e hidrelétrica $(1 g)$ e o atendimento dos limites de fluxos máximos nas linhas de transmissão.

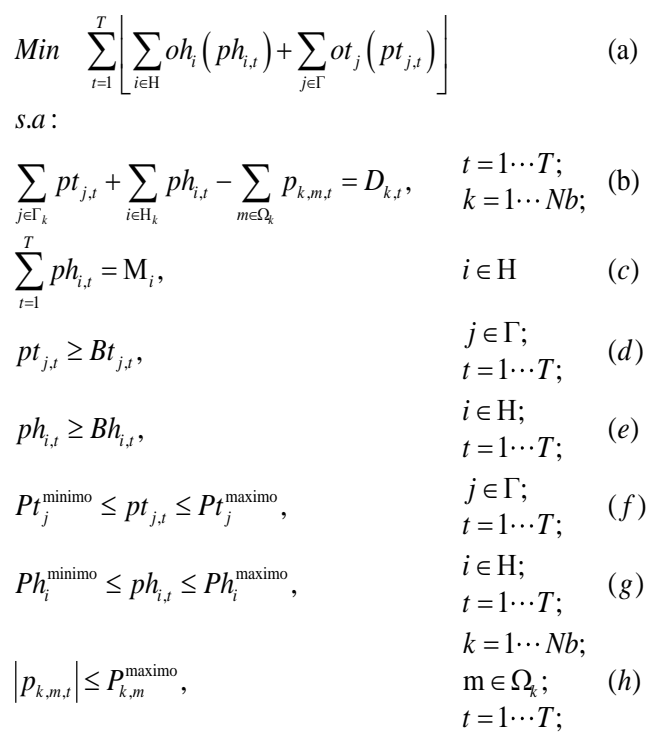

Os custos de oferta de energia das usinas termelétricas $j$ são representados por funções quadráticas (2), cujos parâmetros $B 0_{j}, B 1_{j}, B 2_{j}$ são as ofertas realizadas pelo agente da usina termelétrica, cujas unidades são $\left.\mid \$ / M W^{2}\right\rfloor,[\$ / M W]$ e $[\$]$, respectivamente.

$$
o t_{j}\left(p t_{j, t}\right)=B 2_{j} p t_{j, t}^{2}+B 1_{j} p t_{j, t}+B 0_{j}
$$

Os custos de oferta dos geradores hidrelétricos $i$ são estimados através da expressão mostrada em (3), que são calculados a partir da precificação das perdas relativas ao processo de produção hidráulico de eletricidade, propostas em (Soares, Salmazo, 1997).

$$
o h_{i}\left(p h_{i, t}\right)=p r_{k, t} l_{i}\left(p h_{i, t}\right)
$$

A precificação se dá mediante a multiplicação do preço de equilíbrio da energia $\left(p r_{k, t}\right)$, em cada barra $k$ para cada intervalo de tempo $t$, pela função de perdas hidráulicas mostradas em (4), onde $A 2_{i}, A 1_{i}, A 0_{i}$ representam os parâmetros da curva de perdas para cada hidrelétrica, cuja unidades são em $[1 / M W]$, valor adimensional e $[M W]$, respectivamente.

$$
\operatorname{li}\left(p h_{i, t}\right)=A 2_{i} p h_{i, t}{ }^{2}+A 1_{i} p h_{i, t}+A 0_{i}
$$


A restrição de atendimento de demanda $(1 b)$ é implementada mediante a verificação do atendimento do balanço de potência ativa para todo intervalo de tempo $t$, para cada barra/região do sistema $\mathrm{e}$ determina que o somatório da potência ativa gerada por todas as termelétricas, pertencentes a uma barra/região $\Gamma_{k}$ acrescido do somatório da potência ativa gerada por todas as hidrelétricas $i$ pertencente a uma barra/região $\mathrm{H}_{k}$, decrescido da somatória do fluxo de potência ativa ( $\left.\sum_{m \in \Omega} p_{k, m, t}\right)$ fluindo através das linhas de transmissão que interligam uma barra/região $k$ à sua vizinhança dada por $\mathrm{m} \in \Omega_{\mathrm{k}}$, devem se igualar à demanda energética $\left(D_{k, t}\right)$, dados em $[M W]$.

O fluxo de potência ativa $\left(p_{k, m, t}\right)$, dado em $[M W]$, presente nas restrições $(1 b)$ e $(1 h)$, é dado pelo fluxo ativo de potência linearizado e pelas perdas ôhmicas quadráticas nas linhas de transmissão, conforme mostrado em (5)

$$
p_{k, m, t}=\frac{\left(\theta_{k, t}-\theta_{\mathrm{m}, t}\right)}{X_{k m}}+\frac{G_{k m}\left(\theta_{k, t}-\theta_{\mathrm{m}, t}\right)^{2}}{2}
$$

Em que:

$X_{k m}$ : reatância elétrica da linha de transmissão entre barra/região $k$ e a barra/região $m$ dada em [ohms]; $G_{k m}$ : condutância elétrica da linha de transmissão entre barra/região $k$ e a barra/região $m$ dado em $[S]$; $\theta_{k, t}$ : ângulo da barra/região $k$ no intervalo de tempo $t$ dada em $[\mathrm{rad}]$;

A restrição (1c) estabelece metas energéticas diárias a serem cumpridas pelos geradores hidrelétricos $\left(M_{i}\right)$. Essas metas são dadas em $[M W]$, e são fornecidas pela solução de problemas de planejamento de longo/médio e acoplam o problema hidráulico através de uma variável elétrica. É importante notar que o risco de déficit é intrinsecamente mitigado por esse tipo de restrição.

A restrição $(1 d)$ e $(1 e)$ estabelecem que a potência gerada proveniente dos agentes geradores hidrelétricos $i$ e termelétricos $j$, no intervalo de tempo $t$, devem ser maior ou igual ao montante dos contratos bilaterais firmados entre esses agentes geradores e seus consumidores, dados em $[M W]$.

As restrições ( $1 f)$ e $(1 g)$ determinam que as usinas termelétricas e hidrelétricas, respectivamente, operem dentro de seus limites nominais de geração de potência ativa.

As restrições (1h) dizem respeito a representação da transmissão no modelo LMSHRT, e determina que o valor absoluto do fluxo de potência ativa nas linhas de transmissão, que fluem da barra/região $k$ para barra/região adjacente $m$, para todo tempo $t$, devem ser iguais ou inferiores ao limite máximo da capacidade de transmissão das linhas, denotado por $\left(P_{k, m}^{\text {maximo }}\right)$ e dado em $[M W]$. É importante salientar que no tocante às perdas ôhmicas nas linhas de transmissão vários trabalhos, entre eles (Motto,
Galiana, Conejo, Arroyo, 2002) modelam estas perdas através de linearizações por partes, resultando em um nível de simplificação ainda maior do que a modelagem quadrática das perdas ôhmicas adotada no cálculo do fluxo de potência ativa adotados nas restrições $(1 b)$ e $(1 h)$ deste trabalho.

Em relação ao cálculo da função de custo de oferta das hidrelétricas, este depende da definição dos preços de equilíbrio de mercado mostrado em (6) para cada barra/região $\mathrm{k}$ em cada intervalo de tempo $\mathrm{t}$, os quais são funções dos multiplicadores de Lagrange $\left(\lambda_{k, t}\right)$ associados às restrições de atendimento de demanda em $(1 b)$

$$
p r_{k, t}=-\lambda 1_{k, t}
$$

Sabe-se da teoria marginalista (Schweppe, Caramanis, Tabors, Bohn, 1988) que o multiplicador de Lagrange da restrição de atendimento de demanda, denotado por $-\lambda_{k, t}$, representa o preço de equilíbrio de mercado da energia elétrica para cada barra $k$ em cada intervalo de tempo $t$, e devido a abordagem adotada para o cálculo do preço de equilíbrio, a função objetivo que deve ser minimizada depende de $\lambda_{k, t}$.

No entanto, como seu o valor está condicionado ao multiplicador de Lagrange da restrição de atendimento de demanda $(1 b)$, que só pode ser obtido a medida em que o problema de otimização (1) vai sendo resolvido, o problema de definição dos preços ( $p r_{k, t}$ ) deve passar por um processo recursivo, onde é fixado um determinado valor inicial para $p r_{k, t}$, que é atualizado iterativamente, fazendo-se $p r_{k, t}=-\lambda_{k, t}$, até que o processo se estabilize com uma determinada precisão $\varepsilon$. O algoritmo para a obtenção de $p r_{k, t}$ é mostrado na Figura 1.

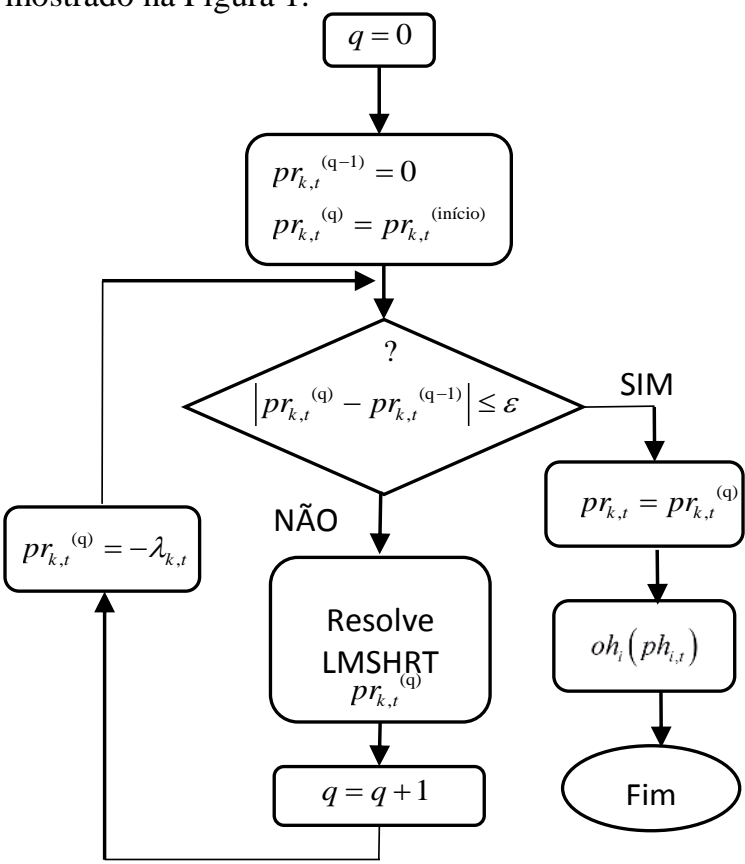

Figura 1 - Processo recursivo para a obtenção do preço de equilíbrio de mercado 
No entanto, a estratégia adotada para o cálculo de $p r_{k, t}$ consiste em atualizar $p r_{k, t}^{(q)}=-\lambda_{k, t}^{(q)}$ de forma iterativa durante o processo de otimização (onde $q$ é a iteração do processo de otimização). Essa forma mais simples e computacionalmente mais eficiente de cálculo produz os mesmos valores obtidos pela estratégia descrita na Figura 1.

\section{Dados do Sistema Teste}

Para a simulação e análise dos resultados foi utilizado um sistema teste com 30 barras extraído de (Christie, 1993) e adaptado com a inclusão de 4 usinas hidrelétricas conectadas nas barras $1,8,11$ e 13 (Jurumirim, Ourinhos, Piraju e Chavantes, respectivamente) e 2 usinas termelétricas conectadas nas barras 5 e 2 (Termelétrica 1 e Termelétrica 2, respectivamente). Outros sistemas foram estudados os quais não são aqui descritos por razões de extensão do artigo. Os dados relativos às usinas termelétricas e hidrelétricas foram extraídos de (Vergílio, 2011). Na Tabela 1 mostram-se as metas energéticas das usinas hidrelétricas.

Tabela 1. Dados de meta energéticas das usinas hidrelétricas.

\begin{tabular}{|c|c|c|c|c|}
\hline Usina & $\begin{array}{c}\text { Jurumi- } \\
\text { rim }\end{array}$ & Piraju & Chavantes & Ourinhos \\
\hline Meta & 1349 & 1204,2 & 5807,7 & 673,16 \\
\hline
\end{tabular}

Na Tabela 2 apresenta-se uma amostra percentual de um perfil típico de carga diária extraído do pacote computacional ANAREDE. Na Tabela 3 são apresentados os dados de demanda (Pcon) por barra do sistema teste IEEE 30 barras extraído de (Christie, 1993). Assim de posse da Tabela 2 e 3 e da expressão (7), são calculados os coeficientes $\alpha_{t}$ para cada intervalo de tempo $t$, que multiplicados pelo dados de potência consumida por barra da Tabela 3, resulta em um perfil típico de carga do sistema interligado nacional (SIN) para cada barra, em 24 intervalos de tempo, de forma que a carga da Tabela 3 fique distribuída conforme um perfil típico de demanda de carga do sistema brasileiro para cada barra do sistema em base horária, dados em $[M W]$.

Tabela 2. Perfil de demanda total em base horária.

\begin{tabular}{|c|c|c|c|c|c|}
\hline Hora & Demanda & Hora & Demanda & Hora & Demanda \\
\hline 1 & 511 & 9 & 518 & 17 & 539 \\
\hline 2 & 483 & 10 & 539 & 18 & 574 \\
\hline 3 & 469 & 11 & 553 & 19 & 700 \\
\hline 4 & 462 & 12 & 546 & 20 & 672 \\
\hline 5 & 455 & 13 & 539 & 21 & 637 \\
\hline 6 & 469 & 14 & 535,5 & 22 & 602 \\
\hline 7 & 476 & 15 & 534,1 & 23 & 567 \\
\hline 8 & 483 & 16 & 533,4 & 24 & 525 \\
\hline
\end{tabular}

$$
\alpha_{t}=\frac{\sum_{k=1}^{N b} \text { Demand }_{k, t}}{\sum_{K=1}^{N b} \text { Pcon }_{k}}
$$

Tabela 3. Potência consumida por barra.

\begin{tabular}{|c|c|c|c|c|c|c|c|}
\hline Barra & Pcon & Barra & Pcon & Barra & Pcon & Barra & Pcon \\
\hline 1 & 0,0 & 9 & 0,0 & 17 & 9,0 & 25 & 0,0 \\
\hline 2 & 21,7 & 10 & 5,8 & 18 & 3,2 & 26 & 3,5 \\
\hline 3 & 2,4 & 11 & 0,0 & 19 & 9,5 & 27 & 0,0 \\
\hline 4 & 7,6 & 12 & 11,2 & 20 & 2,2 & 28 & 0,0 \\
\hline 5 & 94,2 & 13 & 0,0 & 21 & 17,5 & 29 & 2,4 \\
\hline 6 & 0,0 & 14 & 6,2 & 22 & 0,0 & 30 & 10,6 \\
\hline 7 & 22,8 & 15 & 8,2 & 23 & 3,2 & & \\
\hline 8 & 30,0 & 16 & 3,5 & 24 & 8,7 & & \\
\hline
\end{tabular}

Na Tabela 4 mostra-se o montante de energia bilateral contratada em cada barra de geração do sistema para cada intervalo de tempo, dado em $[M W]$

Tabela 4. Energia contratada das usinas no mercado bilateral.

\begin{tabular}{|c|c|c|c|c|c|c|}
\hline Hora & $\begin{array}{c}\text { Barra } \\
5\end{array}$ & $\begin{array}{c}\text { Barra } \\
2\end{array}$ & $\begin{array}{c}\text { Barra } \\
1\end{array}$ & Barra 11 & Barra 13 & Barra 8 \\
\hline 1 & 0 & 18 & 23 & 28 & 82 & 9 \\
\hline 2 & 0 & 18 & 22 & 26 & 77 & 8 \\
\hline 3 & 0 & 30 & 21 & 25 & 75 & 8 \\
\hline 4 & 0 & 28 & 21 & 25 & 74 & 8 \\
\hline 5 & 20 & 28 & 21 & 25 & 73 & 8 \\
\hline 6 & 20 & 38 & 21 & 25 & 75 & 8 \\
\hline 7 & 25 & 40 & 22 & 26 & 76 & 8 \\
\hline 8 & 40 & 35 & 22 & 26 & 77 & 8 \\
\hline 9 & 40 & 32 & 23 & 28 & 83 & 9 \\
\hline 10 & 20 & 34 & 24 & 29 & 86 & 9 \\
\hline 11 & 30 & 35 & 25 & 30 & 88 & 9 \\
\hline 12 & 40 & 35 & 25 & 30 & 87 & 9 \\
\hline 13 & 30 & 34 & 24 & 29 & 86 & 9 \\
\hline 14 & 30 & 34 & 24 & 29 & 86 & 9 \\
\hline 15 & 30 & 34 & 24 & 29 & 85 & 9 \\
\hline 16 & 30 & 34 & 24 & 29 & 85 & 9 \\
\hline 17 & 30 & 34 & 24 & 29 & 86 & 9 \\
\hline 18 & 30 & 40 & 26 & 31 & 92 & 10 \\
\hline 19 & 30 & 40 & 32 & 38 & 112 & 12 \\
\hline 20 & 30 & 40 & 30 & 36 & 108 & 11 \\
\hline 21 & 0 & 40 & 29 & 34 & 102 & 11 \\
\hline 22 & 0 & 39 & 27 & 33 & 96 & 10 \\
\hline 23 & 0 & 25 & 26 & 31 & 91 & 10 \\
\hline 24 & 0 & 18 & 24 & 28 & 84 & 9 \\
\hline & & & & & & \\
\hline
\end{tabular}

Na Tabela 5 são apresentados os limites de capacidade máxima de transmissão do fluxo de potência ativa nas linhas do sistema (LMax), dados em $[M W]$.

Tabela 5. Capacidade de transmissão do sistema.

\begin{tabular}{|c|c|c|c|c|c|c|c|}
\hline Ramo & LMax & Ramo & LMax & Ramo & LMax & Ramo & LMax \\
\hline 1 & 63,8 & 12 & 4,4 & 23 & 37,4 & 34 & 9,9 \\
\hline 2 & 18,7 & 13 & 63,8 & 24 & 11 & 35 & 12,1 \\
\hline 3 & 4,4 & 14 & 35,2 & 25 & 5,5 & 36 & 25,3 \\
\hline 4 & 12,1 & 15 & 121 & 26 & 37,4 & 37 & 17,6 \\
\hline 5 & 73,7 & 16 & 363 & 27 & 38,5 & 38 & 19,8 \\
\hline 6 & 26,4 & 17 & 38,5 & 28 & 17,6 & 39 & 11 \\
\hline 7 & 103,4 & 18 & 106,7 & 29 & 9,9 & 40 & 2,2 \\
\hline 8 & 26,4 & 19 & 78,1 & 30 & 50,6 & 41 & 27,5 \\
\hline 9 & 88 & 20 & 20,9 & 31 & 7,7 & & \\
\hline 10 & 46,2 & 21 & 64,9 & 32 & 40,7 & & \\
\hline 11 & 29,7 & 22 & 48,4 & 33 & 23,1 & & \\
\hline
\end{tabular}

A partir desses dados foi resolvido o LMHSRT utilizando-se o MPI. A precisão adotada para a convergência do MPI foi de $\varepsilon=1 \times 10^{-6}$ e o método convergiu em uma média de 28 iterações para a simulação sem congestionamento e 39 iterações para o sistema com congestionamento.

\section{Resultados Simulação}

O modelo LMHSRT proposto é aplicado no sistema teste IEEE 30 barras que foi modificado conforme descrito na seção 3. Para evidenciar o efeito que o congestionamento e as perdas nas linhas de 
transmissão provocam na formação de preços nas barras do sistema, são implementados aqui testes para cenários distintos. $\mathrm{O}$ primeiro cenário apresenta $\mathrm{o}$ cálculo dos preços nodais sem congestionamento nas linhas de transmissão e o segundo cenário apresenta o cálculo dos preços nodais com um congestionamento artificialmente forçado em algumas linhas de transmissão do sistema, com o intuito de se observar a influência da transmissão do sistema na formação de preços para cada barra.

Desse modo, no segundo cenário, os limites máximos de fluxo de potência ativa nas linhas de transmissão que conectam as barras 23,24 e 28 às barras $15,22,6$ e 8 , respectivamente, foram reduzidos em $12,5 \%$. O percentual de redução foi o máximo conseguido para as referidas linhas de transmissão do sistema sem que a simulação computacional do sistema se tornasse infactível. As demais linhas de transmissão do sistema não tiveram seus limites máximos de capacidade de transmissão alterados em relação ao limites de capacidade máxima de transmissão apresentados na Tabela 5.

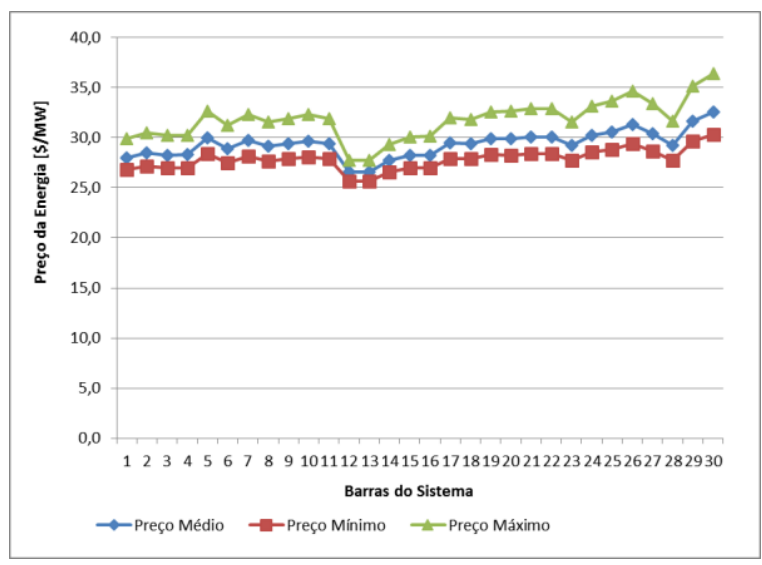

Figura 2 - Preço mínimo, médio e máximo de cada barra sem restrição transmissão

Na Figura 2 são apresentados o comportamento dos preços mínimo, médio e máximo para todas as barras do sistema teste sem congestionamento na transmissão, dados em $[\$ / M W]$. Na Figura 3 são apresentados o comportamento dos preços mínimo, médio e máximo para todas as barras do sistema teste com congestionamento de $12.5 \%$ em algumas linhas, conforme já citado.

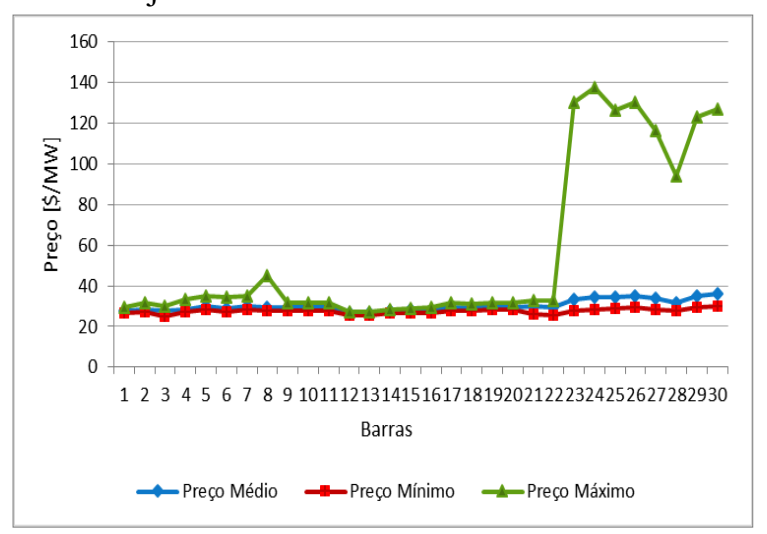

Figura 3 - Preço mínimo, médio e máximo de cada barra com restrição transmissão
É importante destacar que os preços foram calculados em base horária com 24 intervalos de tempo com intuito de se representar um comportamento diário de preços para cada barra do sistema.

Nota-se assim, para o cenário com a transmissão congestionada, uma acentuada elevação de preços, em algumas barras superior a $300 \%$, que denotam uma região de preços diferenciados em relação ao restante do sistema. Notou-se também, que o crescimento dos preços nas barras contidas nessa região se dá nas horas de maior consumo (hora crítica) propiciado pela maior carga decorrente do perfil de demanda do sistema.

Esse fenômeno demonstra claramente a formação de preços regionalizados no sistema, em que o preço da região que sofreu congestionamento em suas linhas de transmissão apresenta um valor médio e máximo maior do que os preços do restante do sistema que não sofreu qualquer congestionamento em suas linhas de transmissão. Obviamente, os consumidores que estão conectados às barras dessa região serão seriamente penalizados com tarifas de energia muito superiores aos consumidores do restante do sistema. Essa situação deve ser evitada, na prática, com políticas de redimensionamento do sistema de transmissão, através da construção de linhas adicionais que descongestionem a transmissão e façam com que as tarifas de energia na região estejam mais próximas daquelas do restante do sistema.

\section{Conclusão}

Este trabalho propõe um novo modelo de leilão com restrições da transmissão para mercados hidrotérmicos. O modelo proposto evita procedimentos e correções ex post para a alocação das perdas e representação dos congestionamentos na transmissão. Os resultados obtidos evidenciam ainda que os congestionamentos na transmissão têm impacto direto na formação de preços de equilíbrio horários por barra no sistema. O modelo permite evidenciar a formação de preços mais elevados em determinadas barras do sistema, e também a formação de preços regionalizados, os quais geralmente ocorrem em situações de congestionamento. $\mathrm{O}$ modelo também identifica áreas do sistema de transmissão que apresentam fator de sub-dimensionamento.

\section{Agradecimentos}

Este trabalho contou com apoio da CAPES Coordenação de Aperfeiçoamento de Pessoal de Nível Superior.

\section{Referências Bibliográficas}

Arroyo, J. M., Conejo, A. J., Galiana, F. D., Motto, A. L., (2002). Network-Constrained Multiperiod Auction for a Pool-Based Electricity Market. 
IEEE Transactions on Power Systems, v. 17, n.2, p. 646-652. DOI: 10.1109/TPWRS.2002.804952

Carrión A. J., Arroyo, J. M., (2006). A Computationally Efficient Mixed-Integer Linear Formulation for the Thermal Unit Commitment Problem. IEEE Transactions on Power Systems, v. 21, n.2, p. 1371-1378. DOI: 10.1109/TPWRS.2006.876672

Christie, R. D., (1993). University of Washington Electrical Engineering. Disponível em: http://www.ee.washington.edu/research/pstca/pf 30/ieee30cdf.txt [Acesso em 06/02/2013].

Conejo, A. J., Arroyo, J. M., (2002). Multiperiod Auction for a Pool-Based Eletricity Market. IEEE Transactions on Power Systems, v. 17, n. 4, p. 1225-1231. DOI: 10.1109/TPWRS.2002.804951

Conejo, A. J., Galiana, F. D., Arroyo, J. M., GarcíaBertrand, R., Chua, C. W., Huneault, M., (2003). Economic inefficiencies and Cross-Subsidies in an Auction-Based Electricity Pool. IEEE Transactions on Power Systems, v. 3, n. 4, p. 221-227. DOI: 10.1109/TPWRS.2002.807118

Fosso, O. B., Gjesvilk, A., Haugstad, A., Mo, B., Wangensteen, I., (1999). Generation Scheduling in a Deregulated System. The Norwegian Case. IEEE Transactions on Power Systems, v. 14. n. 1, p. 75-81. DOI: 10.1109/59.744487

Luh, P. B., Blankson, W. E., Cheng, Y., Yan, J. H., Stern, G. A., Chang, S., Zhao, F., (2006). Payment Cost Minimization Auction for Deregulated Electricity Markets Using Surrogate Optimization. IEEE Transactions on Power Systema, v. 21, n. 2, p. 568-578. DOI: 10.1109/TPWRS.2006.873104

Motto, A. L., Galiana, F. D., Conejo, A. J., Arroyo, J. M., (2002). Network-Constrained Multiperiod Auction for Pool-Based Electricity Market. IEEE Transactions on Power Systems, v. 17, n. 3, p. 646-653. DOI: 10.1109/TPWRS.2002.800951

Schweppe, F. C., Caramanis, M. C., Tabors, R. D., Bohn, R. E., (1988). Spot Pricing of Electricity. Boston: Kluwer Academic Publishers. DOI: 10.1007/978-1-4613-1683-1

Soares, S., Salmazo, C. T., (1997). Minimum loss predispatch model for hydroelectric power system. IEEE Transactions on Power Systems, August, v. 12, n. 3, p. 1220. DOI: $10.1109 / 59.630464$

Vergílio, A. H. B., (2011). Um modelo de prédespacho hidrotérmico para mercados de energia. Bauru: UNESP - Universidade Estadual Paulista. Disponível em:

http://www2.feb.unesp.br/pos/bibliotecavirtual/d ocumento.php?COD=854d6fae5ee42911677c73 $\underline{9 e e 1734486}$ [Acesso em 08/03/2013].

Wright, S., (1997). Primal-dual Interior Point Methods. SIAM . DOI: 10.1137/1.9781611971453 